\title{
Time-dependent neoclassical viscosity
}

\author{
A. L. Garcia-Perciante, J. D. Callen, K. C. Shaing, and C. C. Hegna \\ University of Wisconsin-Madison, Madison, Wisconsin 53706-1687
}

(Received 6 October 2004; accepted 28 February 2005; published online 9 May 2005)

\begin{abstract}
A time-dependent closure for the parallel viscous force is calculated in a bumpy cylinder magnetic field geometry using a Chapman-Enskog-like approach. The calculation is valid for all times and field modulations, and is expressed as a dynamic evolution in time. Two important applications are presented: modification of the frequency-dependent electrical conductivity due to the interaction between trapped and circulating particles, and the parallel flow evolution which can be extended to axisymmetric geometries. ㅇ 2005 American Institute of Physics. [DOI: 10.1063/1.1899159]
\end{abstract}

\section{INTRODUCTION}

Most present day plasma confinement devices operate in the low collisionality "banana" regime where the relevant closure moment is the parallel stress tensor. The parallel viscous force affects the dynamics of flows and is a drive in the momentum equation. In particular, the electron viscous drag in the parallel Ohm's law introduces a modification of the electrical conductivity. Also, in the total momentum balance, the parallel flow is damped by this stress term. The equivalent effect in tokamak geometry produces poloidal flow damping.

The closure for the parallel viscous force has been calculated assuming a steady state. ${ }^{1-4}$ Using the closures obtained in the steady state calculation, the damping rate of the poloidal flow in a toroidal plasma becomes comparable with the ion-ion collision frequency. This conclusion seems to violate the static assumption used to derive the closure. ${ }^{5}$

Various authors have addressed the time-dependent problem ${ }^{6-10}$ both by a variational principle and using an expansion in Cordey eigenfunctions ${ }^{11}$ of the pitch-angle Coulomb collision scattering operator. Flow damping rates are estimated to be of the order of the ion collision frequency times some power of the inverse aspect ratio when a large aspect ratio limit is used. ${ }^{6-10}$

In this work, the dynamic case is approached in a different way. The evolution of the parallel flow is found to be more than a simple exponential and thus cannot be characterized by a single damping rate. Instead of addressing each stage of the evolution separately as a frequency-dependent problem, the main objective here is to describe the time evolution of the parallel viscous force. This responds to the need for a complete picture of the transition to the steady state and the determination of the time scale on which an equilibrium assumption is formally valid. The relaxation of the poloidal flow occurs on a fairly fast time scale (about the ion-ion collision time) but is still relevant to some experiments, for instance, just after a sawtooth $\operatorname{crash}^{12}$ and in microturbulence. ${ }^{10}$

The procedure presented here is based on a ChapmanEnskog-like approach ${ }^{13}$ which is described in Sec. II. In Sec. III the drift kinetic equation (DKE) is stated and adapted to a simplified magnetic field geometry. A perturbation technique that provides a solution to the lowest order DKE is described in Sec. IV and the closure is obtained in Sec. V. Sections VI and VII give two relevant applications of the result: the modification for the parallel electrical conductivity and the evolution of the parallel/poloidal flow, respectively. A summary and concluding remarks are included in Sec. VIII. Some details of the calculation leading to the frequencydependent closure are shown in Appendix A. The Laplacetransform inversions for the closure and the flow evolution are shown in Appendix B. Finally, heat flux effects are considered in Appendix C.

\section{CHAPMAN-ENSKOG-LIKE APPROACH}

In a Chapman-Enskog-like approach, the distribution function of a system is assumed to be a dynamic, flowshifted and heat flow-shifted Maxwellian plus a small (compared with the equilibrium solution) kinetic deviation $F$. In most of this work, heat flux effects will be neglected for simplicity (they are discussed in Appendix C); thus, the distribution function for the system will be assumed to $\mathrm{be}^{14}$

$$
f=f_{M}+F,
$$

where $f_{M}$ is a Maxwellian distribution function in the relative velocity $\mathbf{v}=\mathbf{v}^{\prime}-\mathbf{V}$ :

$$
f_{M}\left(\mathbf{v}^{\prime}, \mathbf{x}, t\right)=n\left(\frac{m}{2 \pi k T}\right)^{3 / 2} \exp \left[-\frac{m\left(\mathbf{v}^{\prime}-\mathbf{V}\right)^{2}}{2 k T}\right] .
$$

Here $n(\mathbf{x}, t)$ is the number density, $\mathbf{V}(\mathbf{x}, t)$ the flow velocity, $T(\mathbf{x}, t)$ the temperature, and $m$ the particle mass.

By the Chapman-Enskog hypothesis, the first three moments of the kinetic distortion vanish and the time dependence of the distribution function is given through the variations in the thermodynamic variables in the Maxwellian. Thus, for the present problem, the Chapman-Enskog ansatz is subject to the constraint conditions

$$
\int d^{3} v F=0, \quad \int d^{3} v \mathbf{v} F=0, \quad \int d^{3} v \frac{m v^{2}}{2} F=0 .
$$

By including the first three moments of the distribution function in the Maxwellian part, the kinetic distortion does not add terms to the density, momentum, and energy balance equations. Neoclassical effects will appear in this formulation through higher order moments of $F$, in particular, 


$$
\Pi=\int d^{3} v m\left(\mathbf{v v}-\frac{1}{3} v^{2} \mathbf{I}\right) F,
$$

which gives rise to the effects that are of concern in this work. The relevant closure which is the focus of this work is the (flux-surface-averaged) parallel viscous force $\left\langle\mathbf{B} \cdot \boldsymbol{\nabla} \cdot \Pi_{\|}\right\rangle$; it will be obtained from the solution of the drift kinetic equation as will be shown in the following section.

\section{DRIFT KINETIC EQUATION}

For a system of charged particles and independent variables $\mathbf{v}, \mathbf{x}$, and $t$, the total time derivative on the left side of the plasma kinetic equation is

$$
\begin{aligned}
\frac{d f}{d t}= & \frac{\partial f}{\partial t}+\mathbf{v} \cdot \boldsymbol{\nabla} f+\left[\frac{q}{m}\left(\mathbf{E}+\frac{1}{c}(\mathbf{V}+\mathbf{v}) \times \mathbf{B}\right)-\frac{\partial \mathbf{V}}{\partial t}-(\mathbf{V}\right. \\
& +\mathbf{v}) \cdot \boldsymbol{\nabla} \mathbf{V}] \cdot \frac{\partial f}{\partial \mathbf{v}} .
\end{aligned}
$$

The distribution function given by Eqs. (1)-(3) can be introduced in Eq. (5) and using the density, momentum, and energy balance equations the total time derivative of the distribution function can be written in terms of $F$. Using a model collision operator that separates the effects on $f_{M}$ and $F$, Wang and Callen ${ }^{13}$ recasted the kinetic equation in a formal gyroaveraged evolution equation for the distortion $F$. The full equation is not written here since only a simplified version of it will be considered. Including heat flux effects in the calculation is straightforward as shown in Appendix C. Thus, neglecting all heat flux terms, Eq. (127) in Ref. 13 reduces to

$$
\begin{aligned}
\frac{\partial F}{\partial t}+ & \left(v_{\|} \mathbf{b}+\mathbf{V}\right) \cdot \nabla F-C(F)=\left[\left(\frac{v_{\perp}^{2}}{2}-v_{\|}^{2}\right) G\right. \\
& \left.+\frac{v_{\|}}{p} \mathbf{b} \cdot \boldsymbol{\nabla} \cdot \Pi_{\|}\right] f_{M},
\end{aligned}
$$

where the "flow drive" term is

$$
G=\frac{m}{T}\left[\mathbf{V} \cdot \boldsymbol{\nabla} \ln B-\frac{1}{B} \mathbf{b} \cdot \boldsymbol{\nabla} \times(\mathbf{B} \times \mathbf{V})+\frac{2}{3} \boldsymbol{\nabla} \cdot \mathbf{V}\right] .
$$

The approximate collision operator we consider is ${ }^{7}$

$$
C(F)=\bar{\nu} \mathcal{L}(F)+\sum_{l} P_{l}\left[C^{l}\left(f_{l}\right)+\frac{l(l+1)}{2} \bar{\nu} f_{l}\right],
$$

where $P_{l}$ are Legendre polynomials with arguments $\left|v_{\|} / v\right|$ $=\sqrt{1-\lambda B}, \lambda \equiv \mu / E$, and $\bar{\nu} \equiv \nu_{\perp} / 2$. The first term in Eq. (8) accounts for pitch-angle scattering with $\mathcal{L}$ being the Lorentz scattering operator. The second term contains the momentum restoring terms (required since $\mathcal{L}$ does not conserve momentum by itself) where

$$
f_{l}=\frac{2 l+1}{4} \sum_{\varsigma} \int_{0}^{1 / B} \frac{d \lambda B}{\sqrt{1-\lambda B}} P_{l}(\sqrt{1-\lambda B}) f .
$$

Since the collision operator in Eq. (8) is expanded in Legendre polynomials it only affects the pitch-angle dependence of the first three harmonics of the distribution func- tion. Because of this and the fact that the full Coulomb collision operator is rotationally symmetric, the speed $v$ can be treated as a parameter in the DKE which simplifies its solution. Also, the series can be truncated at $l=2$ because the higher order terms are smaller in the small mass ratio expansion. ${ }^{15}$ Only the first three terms are required for the proposed collision operator to conserve density, momentum, and energy. ${ }^{16}$

A bumpy cylinder magnetic field model simplifies the calculation of the kinetic distortion $F$ while retaining the effects of pitch angle scattering of particles in and out of trapped space. The functional form of such a field can be written as

$$
B(\ell)=B_{\min }+\Delta B \sin ^{2} \frac{\pi \ell}{L},
$$

where $\Delta B=B_{\max }-B_{\min } \equiv 2 B_{\min } \epsilon$ and $\ell$ is the axial length following the field line as it curves. Within the scale length of interest, which is the periodicity length of the cylinder $L$, only the magnetic field will be allowed to vary, retaining only the $\nabla_{\|} B$ effects. (There are no radial drifts in an axisymmetric bumpy cylinder magnetic field.)

In this simple configuration

$$
\boldsymbol{\nabla} \times(\mathbf{B} \times \mathbf{V})=0 .
$$

Hence, the second term in the drive $G$ is absent. For the last term in Eq. (7), a particle continuity equation is used. For bounce time scales we have

$$
0 \simeq \frac{\partial n}{\partial t}=-n \boldsymbol{\nabla} \cdot \mathbf{V}-\mathbf{V} \cdot \boldsymbol{\nabla} n,
$$

which leads to an incompressible flow to lowest order for density constant along the magnetic field. As a consequence, by noting that

$$
\boldsymbol{\nabla} \cdot \mathbf{V}=(\mathbf{B} \cdot \boldsymbol{\nabla})\left(\frac{V_{\|}}{B}\right)=0,
$$

the incompressibility constraint can then be satisfied by defining a parallel flow variable $V_{\|}(\ell, t) / B(\ell)=U(t)$ on a given magnetic flux surface.

Introducing these simplifications in Eq. (7) and using the relation $v_{\|} \mathbf{b} \cdot \boldsymbol{\nabla}\left(v_{\|} B\right)=\left(v_{\|}^{2}-v_{\perp}^{2} / 2\right) \mathbf{B} \cdot \boldsymbol{\nabla} \ln B$, the flow drive term reduces to

$$
\left(v_{\|}^{2}-\frac{v_{\perp}^{2}}{2}\right) G=\frac{m}{T} v_{\|} \mathbf{b} \cdot \boldsymbol{\nabla}\left(v_{\|} B\right) U .
$$

The DKE can then be written as

$$
\frac{\partial F}{\partial t}+v_{\|} \mathbf{b} \cdot \boldsymbol{\nabla}\left(F+\frac{m}{T} v_{\|} B U f_{M}\right)-C(F)=\frac{v_{\|}}{p} f_{M} \mathbf{b} \cdot \boldsymbol{\nabla} \cdot \Pi_{\|} .
$$

Thus, the dynamic evolution of the kinetic distortion is affected by spatial variations of both the kinetic distortion and the free streaming flow of the circulating particles, and collisions that can drive particles through a perpendicular diffusion process into or out of the trapped region of velocity space. The parallel viscous force is a source for the evolution 
of the unknown distribution $F$ and hence will be present in the solution. However, the Chapman-Enskog constraints given in Eq. (3) will allow an expression to be obtained for $\left\langle\mathbf{B} \cdot \boldsymbol{\nabla} \cdot \Pi_{\|}\right\rangle$that has no explicit dependence on $F$.

\section{LOWEST ORDER SOLUTION FOR $\boldsymbol{F}$}

Equation (15) is solved by a standard perturbation technique in the low collisionality banana regime where trapped particles can complete their orbits before being scattered by collisions. Then, the parameter $\nu_{*}=\nu / \epsilon^{3 / 2} \omega_{b}$ is small and will be used as an expansion parameter for $F$. In defining $\nu_{*}, \nu$ is the collision frequency and $\omega_{b}$ is the bounce frequency. The kinetic distortion is then expanded as follows

$$
F=F_{0}+\nu_{*} F_{1}+\cdots .
$$

Assuming the time derivative is of order $\nu_{*}$, the lowest order DKE is

$$
v_{\|} \mathbf{b} \cdot \nabla\left(F+\frac{m}{T} v_{\|} B U f_{M}\right)=0 .
$$

Since for the model magnetic field considered $\mathbf{b} \cdot \boldsymbol{\nabla}=\partial / \partial \ell$, the term in brackets cannot depend on $\ell$. Thus, the lowest order distortion is given by

$$
F_{0}=-\frac{m}{T} v_{\|} B U f_{M}+g(v, \lambda, \varsigma, t)
$$

Here, $g$ is an integration "constant," which is a function of all the variables of the system other than the length $\ell$ over which the integration was performed. The first term in Eq. (18) represents the free streaming part of $F$ while the second term is a collisional correction. The variable $s$ depends on the direction in which the particles circulate and is defined as $\mathrm{s}=v_{\|} /\left|v_{\|}\right|$.

To next order in $\nu_{*}$ the DKE is

$$
\frac{\partial F_{0}}{\partial t}+v_{\|} \mathbf{b} \cdot \nabla F_{1}-C\left(F_{0}\right)=v_{\|} \frac{1}{p} \mathbf{b} \cdot \nabla \cdot \Pi_{\|} f_{M} .
$$

Since $v_{\|} \mathbf{b} \cdot \nabla F_{1}=v_{\|} \partial F_{1} / \partial \ell$, application of the bounceaveraging integral

$$
\oint \frac{d \ell}{v_{\|}}=\left\{\begin{array}{l}
\int_{0}^{L} d \ell / v_{\|}, \quad \text { untrapped particles } \\
\sum_{\varsigma} \int_{-\ell_{c}}^{\ell_{c}} d \ell /\left|v_{\|}\right|, \quad \text { trapped particles }
\end{array}\right.
$$

annihilates the first-order distortion in Eq. (19). Here $\pm \ell_{c}$ are the turning points of the closed, trapped particle orbits where $v_{\|} \rightarrow 0$. Thus, it is not necessary to solve for $F_{1}$ since the bounce-averaged first-order equation provides a constraint that can be solved for the integration constant in the lowest order solution.

For trapped particles, to take account of density conservation at the tip of the bounce orbits we must have $g_{t}(s)$ $=-g_{t}(-\varsigma)$. Then, for $g_{t}$ even, we have $g_{t}=0$ at $\ell_{c}$. Since $g$ does not depend on $\ell, g_{t}=0$ for all values of $\ell$.

For circulating particles, the bounce-averaged complete first-order DKE yields a differential equation for $g_{c}$. When a Laplace transform is taken, both energy (speed) and fre- quency can be treated as parameters. The resulting equation, which can be solved for the pitch-angle dependence in $\hat{g}_{c}$, is

$$
\begin{aligned}
& -i \omega \frac{1}{2}\left\langle\frac{B}{\sqrt{1-\lambda B}}\right\rangle \hat{g}_{c}-\bar{\nu} \frac{\partial}{\partial \lambda} \lambda\langle\sqrt{1-\lambda B}\rangle \frac{\partial \hat{g}_{c}}{\partial \lambda} \\
& =\frac{s v}{2} \hat{S}(v, \lambda, \omega) .
\end{aligned}
$$

Hats denote Laplace-transformed quantities with transform variable $-i \omega$ and the flux-surface-average is

$$
\langle A\rangle \equiv \oint \frac{d \ell}{B} A / \oint \frac{d \ell}{B} .
$$

In Eq. (21) $\lambda=2 \mu / v^{2}$ is the pitch-angle variable and the drives and initial conditions are included in the source term:

$$
\begin{aligned}
\hat{S}(v, \lambda, \omega) \equiv & \frac{1}{p}\left\langle\widehat{\mathbf{B} \cdot \boldsymbol{\nabla} \cdot \Pi_{\|}}\right\rangle f_{M}-\frac{m}{T}\left\langle B^{2}\right\rangle f_{M}\left(i \omega \hat{U}+U_{0}\right) \\
& +\frac{1}{v}\left\langle B^{2}\right\rangle\left[C^{1}(\hat{V})+\bar{\nu} \hat{V}\right]+f_{M}\left\langle\frac{B}{v_{\|}}\right\rangle g_{0}(\lambda) .
\end{aligned}
$$

The flow-like variable $\hat{V}(\omega, v)$ is defined as

$$
\hat{V}(\omega, v) \equiv \frac{3}{4} \sum_{\varsigma} \varsigma \int_{0}^{\lambda_{c}} d \lambda \hat{g}_{c}
$$

and $U_{0} \equiv U(t=0)$. The speed dependence in $g_{c}(\lambda, v, t=0)$ is arbitrary since the collision operator only operates on the pitch-angle variable. Thus, it is conveniently chosen to be separable in $v, \lambda$ as

$$
g_{c}(\lambda, v, t=0) \sim \frac{m}{T} f_{M}(v) g_{0}(\lambda)
$$

to match the rest of the terms in the source. ${ }^{9}$ Note that the $\lambda$ dependence of the source is only through the initial pitchangle structure of the collisional correction $g_{0}(\lambda)$.

Morris et al. ${ }^{9}$ were the first to recognize that this should be treated as an initial value problem. The collisional diffusion into trapped space of a distribution of untrapped particles will depend strongly (at least for early times) on how close the initial distribution is located relative to the boundary between the two types of particles. An initial distribution of particles is expected to damp more rapidly if it is peaked close to $\lambda_{c}$ than if particles are introduced far away from the trapped-circulating boundary, since then the portion of phase space they have to diffuse through is larger.

Speed and pitch-angle variables can now be separated using an eigenfunction expansion for the pitch-angle operator on the left side of Eq. (21). The function $\hat{g}_{c}$ is projected in Cordey eigenfunctions as was done in Refs. 7-9:

$$
\hat{g}_{c}=\frac{\varsigma v}{2} \sum_{n=1}^{\infty} \hat{Y}_{n}(v, i \omega) \Lambda_{n}(\lambda) .
$$

The $\lambda$-dependent functions $\Lambda_{n}$ are eigenfunctions of the ordinary differential equation 


$$
\frac{d}{d \lambda} \lambda\langle\sqrt{1-\lambda B}\rangle \frac{d \Lambda_{n}}{d \lambda}=\kappa_{n} \frac{d}{d \lambda}\langle\sqrt{1-\lambda B}\rangle \Lambda_{n},
$$

where $\kappa_{n}$ are the eigenvalues and the orthogonality condition is given by

$$
\int \Lambda_{n} \Lambda_{m} \frac{\partial\langle\sqrt{1-\lambda B}\rangle}{\partial \lambda} d \lambda=\delta_{n m} \int \Lambda_{n}^{2} \frac{\partial\langle\sqrt{1-\lambda B}\rangle}{\partial \lambda} d \lambda,
$$

in which the integration is performed over circulating particle velocity space $0<\lambda<\lambda_{c}$.

Using Eqs. (26) and (27), one can solve for the speedand frequency-dependent coefficients. The integration constant in the solution for $F$ can then be expressed in frequency space as

$$
\begin{aligned}
\hat{g}_{c}= & -\frac{\varsigma}{2} \sum_{n=1}^{\infty}\left[\frac{\eta_{n}}{\hat{f}_{c}}\left(\hat{V}\left\langle B^{2}\right\rangle-\left\langle B^{2}\right\rangle^{1 / 2} f_{M} \frac{v}{\bar{\nu}} \frac{m}{T} \hat{f}_{g}\right)\right. \\
& \left.+\frac{m}{T} \frac{v}{\nu} f_{M} \alpha_{n}\right] \frac{\Lambda_{n}}{\kappa_{n}-i \omega / \bar{\nu}} .
\end{aligned}
$$

The fraction of circulating particles is defined here as ${ }^{8}$

$$
\hat{f}_{c} \equiv \hat{f}_{c}(\omega, v)=\sum_{n=1}^{\infty} \frac{\gamma_{n}}{\kappa_{n}-i \omega / \bar{\nu}},
$$

and we analogously defined

$$
\hat{f}_{g} \equiv \hat{f}_{g}(\omega, v)=\sum_{n=1}^{\infty} \frac{\chi_{n}}{\kappa_{n}-i \omega / \bar{\nu}},
$$

where all the coefficients in the sums are defined in Appendix A.

The viscous force, being a source in the differential equation for $\hat{g}_{c}$ [Eq. (21)], is part of its solution through the variable $\hat{V}$. However, the Chapman-Enskog constraint that the momentum moment of the small distortion from the Maxwellian vanishes can be used to relate $\hat{V}$ with the parallel flow $\hat{U}$. Once this relation is determined, Eqs. (24) and (28) can be combined to yield an expression for $\left\langle\widehat{\mathbf{B} \cdot \nabla \cdot \Pi_{\|}}\right\rangle$in terms of the flow $\hat{U}$.

\section{DYNAMIC CLOSURE FOR $\left\langle\overline{\mathrm{B} \cdot \nabla \cdot \Pi_{\|}}\right\rangle$}

The solution for the kinetic correction $\hat{g}_{c}$ obtained in the preceding section still depends on the unknown $\left\langle\widehat{\mathbf{B} \cdot \nabla \cdot \Pi_{\|}}\right\rangle$ through the variable $\hat{V}$. The specific relation is given by Eq. (A8) in Appendix A. However, the Chapman-Enskog ansatz given in Eq. (3) can be used to express $\hat{V}$ in terms of $\hat{U}$. Using this relation and the momentum conserving property of the collision operator, the parallel viscous force can be expressed in terms of the parallel flow variable $\hat{U}$ and the initial conditions. The procedure is similar to that in Ref. 8 and is carried out in some detail in Appendix A. (An alternate, simpler procedure is to use only the pitch-angle part of the Chapman-Enskog momentum constraint together with the Laguerre polynomial expansion of $\hat{V}$ in the laboratory frame where the momentum-conserving properties of the collision operator are not needed. But it is not obvious in such a procedure why only the pitch-angle part of the Chapman-Enskog momentum constraint is used.)

The closure that is obtained can be written as

$$
\left\langle\widehat{\mathbf{B} \cdot \boldsymbol{\nabla} \cdot \Pi_{\|}}\right\rangle=n m\left\langle B^{2}\right\rangle\left[\hat{U} \hat{v}(\omega)+U_{0}+\hat{Y}(\omega)\right],
$$

which coincides with the result in Ref. 8 if initial conditions are neglected. The first term shows the usual proportionality between the viscous force and the parallel flow. The coefficient $\hat{v}(\omega)$ is defined as

$$
\hat{v}(\omega)=\frac{m}{T} \int d^{3} v \bar{\nu} \frac{v^{2}}{3} \frac{f_{M}}{n} \frac{\hat{f}_{t}}{\hat{f}_{c}},
$$

where the fraction of trapped particles is

$$
\hat{f}_{t}=1-\left(1-\frac{i \omega}{\bar{\nu}}\right) \hat{f}_{c} .
$$

The initial pitch-angle distribution is contained in the last term which is defined as

$$
\hat{\Upsilon}(\omega)=\frac{m}{T} \int d^{3} v \frac{v^{2}}{3} \frac{f_{M}}{n} \frac{\hat{f}_{g}}{\hat{f}_{c}} .
$$

Note that for long times, when initial conditions are completely damped and can thus be neglected, one can use the result ${ }^{8}$

$$
f_{c} \equiv \hat{f}_{c}(\omega=0)=\sum \frac{\gamma_{n}}{\kappa_{n}}=\frac{3\left\langle B^{2}\right\rangle}{4} \int_{0}^{\lambda_{c}} \frac{\lambda d \lambda}{\langle\sqrt{1-\lambda B}\rangle},
$$

and the steady state limit of Eq. (31) yields the standard result

$$
\left\langle\mathbf{B} \cdot \boldsymbol{\nabla} \cdot \Pi_{\|}\right\rangle=n m \mu\left\langle B^{2}\right\rangle U .
$$

Here, the viscosity coefficient $\mu=v(0)$ is defined (for electrons) as ${ }^{17}$

$$
\mu_{e}=\frac{m_{e}}{T_{e}} \int d^{3} v \bar{\nu} \frac{v^{2}}{3} \frac{f_{M}}{n_{e}}=[Z+\sqrt{2}-\ln (1+\sqrt{2})] \frac{f_{t}}{f_{c}} \nu_{e},
$$

where $f_{t} \equiv 1-f_{c}$.

Equation (31) is valid for any time scale and $\epsilon$ (or aspect ratio), since no approximations have been introduced so far. From it, a damping rate can be estimated numerically. For an explicit time-dependent expression, neither an analytical nor numerical Laplace inverse transform are trivial to perform since the expression involves infinite sums of terms that depend on integrals over the (numerically generated) eigenfunctions. Moreover, some of these infinite sums reside in the denominator and thus should be calculated to high accuracy if all relevant poles of the response are to be found.

An analytical inversion of the Laplace transform in general is not obtainable. However, for small field variations $\left(\Delta B \ll B_{\min }\right)$ an analytical solution can be obtained through a power series expansion in the small parameter $\sqrt{\epsilon}$. In order to make such an expansion, we invoke the ordering $f_{t} \sim \sqrt{\epsilon}$ $\ll 1$ used by various authors ${ }^{4,8}$ in both dynamic and static situations. The relevant factors to be inverted are $\hat{f}_{t} / \hat{f}_{c}$ and $\hat{f}_{g} / \hat{f}_{c}$, for which we propose the following expansions: 


$$
\begin{aligned}
& \frac{\hat{f}_{t}}{\hat{f}_{c}} \simeq\left(1-\frac{i \omega}{\bar{\nu}}\right) \hat{f}_{t}+\left(1-\frac{i \omega}{\bar{\nu}}\right) \hat{f}_{t}^{2}+\cdots, \\
& \frac{\hat{f}_{g}}{\hat{f}_{c}} \simeq\left(1-\frac{i \omega}{\bar{\nu}}\right)\left(1+\hat{f}_{t}\right) \hat{f}_{g}+\cdots .
\end{aligned}
$$

Introducing Eqs. (38) and (39) in the closure given by Eq. (31), a much simpler expression for the parallel viscous force is obtained and the Laplace transform can be inverted term by term. In particular, to lowest order in $\hat{f}_{t}$ we have

$$
\begin{aligned}
\left\langle\mathbf{B} \cdot \boldsymbol{\nabla} \cdot \Pi_{\|}\right\rangle \simeq & n m\left\langle B^{2}\right\rangle \times \frac{m}{T} \int d^{3} v \frac{v^{2}}{3} \frac{f_{M}}{n} \bar{\nu} L^{-1}\{(1 \\
& \left.\left.-\frac{i \omega}{\bar{\nu}}\right) \hat{f}_{t} \hat{U}\right\},
\end{aligned}
$$

in which $L^{-1}$ is the inverse Laplace transform and initial conditions have been neglected for simplicity but can be easily introduced using Eq. (B5). After calculating the inverse Laplace transform of the term in curly brackets in Eq. (40) (see Appendix B), the time-dependent closure for the parallel viscous force in this small $\epsilon$ limit is

$$
\begin{aligned}
\left\langle\mathbf{B} \cdot \boldsymbol{\nabla} \cdot \Pi_{\|}\right\rangle= & \left\langle B^{2}\right\rangle m n \int d^{3} v \frac{v^{2}}{3} \frac{f_{M}}{n} \bar{\nu} \times \frac{m}{T}\left\{U(t)\left(1-f_{c}\right)\right. \\
& +\frac{1}{\bar{\nu}} \frac{\partial U(t)}{\partial t}\left(1-\sum \gamma_{n}\right) \\
& \left.+\sum \frac{\gamma_{n}}{\kappa_{n}}\left(\kappa_{n}-1\right)^{2} \int_{0}^{t} \frac{d U}{d \tau} e^{-\bar{\nu} \kappa_{n}(t-\tau)} d \tau\right\} .
\end{aligned}
$$

This equation exhibits the explicit behavior in time of $\left\langle\mathbf{B} \cdot \boldsymbol{\nabla} \cdot \Pi_{\|}\right\rangle$. The first term is proportional to the parallel flow and will be dominant in the long time asymptotic limit. The second term contains the time variation of the flow and is important only for times of the order of $1 / \bar{\nu}$. The last term contains the time history through the convolution integral of the time-dependent trapped particle fraction and the intrinsic time dependence of the flow of circulating particles. To higher order in this expansion, this last term develops a series in powers of $\bar{\nu} t$ inside the (convolution) time integral. For estimates of the coefficients in Eq. (41) see Eqs. (51)-(54) below.

\section{ELECTRICAL CONDUCTIVITY}

To obtain the modification of the electrical conductivity caused by the parallel viscous force $\left\langle\mathbf{B} \cdot \boldsymbol{\nabla} \cdot \Pi_{\|}\right\rangle$, the bounceaveraged parallel component of the momentum equation for electrons is considered:

$$
\frac{\partial}{\partial t}\left\langle J_{\|} B\right\rangle=\frac{n_{e} e^{2}}{m_{e}}\left\langle E_{\|} B\right\rangle+\frac{e}{m_{e}}\left\langle\mathbf{B} \cdot \boldsymbol{\nabla} \cdot \Pi_{\| e}\right\rangle-\nu_{e}\left\langle J_{\|} B\right\rangle .
$$

Calculating the Laplace tranform on both sides of Eq. (42) and introducing the frequency-dependent closure given by Eq. (31) yields an $\omega$-dependent electrical conductivity:

$$
\hat{\sigma}(\omega)=\frac{n_{e} e^{2}}{m_{e} \nu_{e}} \frac{1}{\hat{\alpha}_{e}(\omega)},
$$

where

$$
\hat{\alpha}_{e}(\omega)=1+\frac{1}{\nu_{e}}[\hat{v}(\omega)-i \omega]
$$

and initial conditions have been neglected for simplicity.

The dynamic conductivity in Eq. (43) is valid for any frequency (time) and magnetic field modulation. Once again, the analytical process cannot be carried out further. However, a numerical computation could give the frequency dependence and upon taking the inverse Laplace transform, the time evolution of $\sigma$ to some appropriate accuracy.

To obtain the static limit, we have

$$
\alpha_{e}(0)=1+\mu_{e} / \nu_{e} .
$$

The fraction of trapped and circulating particles can be estimated for $\sqrt{\epsilon} \ll 1$ as $f_{t} \simeq 1.46 \sqrt{\epsilon}$. $^{4}$ Considering the value for $\mu_{e}\left(\simeq 1.53 \nu_{e} f_{t} / f_{c}\right.$ for hydrogenic ions $)$ in the small $\sqrt{\epsilon}$ approximation, the usual correction to the static electrical conductivity is obtained:

$$
\sigma=\frac{n_{e} e^{2} / m_{e} \nu_{e}}{1+\mu_{e} / \nu_{e}}=\frac{n_{e} e^{2}}{m_{e} \nu_{e}} \frac{1}{1+2.24 \sqrt{\Delta B / 2 B_{\min }}} .
$$
of

For $\omega \neq 0$ we consider the low and high frequency limits

$$
\hat{\alpha}_{e}(\omega) \simeq 1+\frac{\hat{f}_{t}\left(\omega, \nu_{e}\right)}{\hat{f}_{c}\left(\omega, \nu_{e}\right)}-\frac{i \omega}{\nu_{e}},
$$

where we are considering $\bar{\nu}=\nu_{e}$ for simplicity. Using the expansion in Eq. (38), for $\omega \ll \nu_{e}$ we obtain

$$
\hat{\alpha}_{e}(\omega) \simeq 1+f_{t}-\frac{\omega^{2}}{\nu_{e}^{2}}\left(2 \sum \frac{\gamma_{n}}{\kappa_{n}^{2}}-f_{c}\right)-\frac{i \omega}{\nu_{e}}\left(2 f_{t}+\sum \frac{\gamma_{n}}{\kappa_{n}^{2}}\right) .
$$

Thus, for small $\omega$ the real part of the conductivity will decay from the static limit as $-\left(\omega / \nu_{e}\right)^{2}$ while the imaginary ("reactive") part grows as $\omega / \nu_{e}$. The sums in Eq. (48) can be estimated using the small $\epsilon$ approximation for the eigenfunctions in terms of Legendre functions given in Ref. 11. That is, since in the $\epsilon=0$ case the eigenfunctions are Legendre polynomials, one can consider for small $\epsilon$ (Ref. 11)

$$
\Lambda_{n} \sim P_{\nu_{n}}, \quad \kappa_{n} \sim \nu_{n}\left(\nu_{n}+1\right),
$$

where the index $\nu_{n}$ is an integer plus a small correction proportional to $\sqrt{\epsilon}$ :

$$
\nu_{n}=n+\sqrt{2 \epsilon} \frac{4}{\pi} \frac{\Gamma^{2}(1+n / 2)}{\Gamma^{2}(1 / 2+n / 2)} .
$$

In this approximation, the relevant sums can be obtained and are shown below:

$$
\begin{aligned}
& \sum \gamma_{n} \simeq 1-2.93 \epsilon^{3 / 2}+\mathcal{O}\left(\epsilon^{2}\right), \\
& \sum \gamma_{n} \kappa_{n} \simeq 1+1.48 \sqrt{\epsilon}+\mathcal{O}(\epsilon),
\end{aligned}
$$




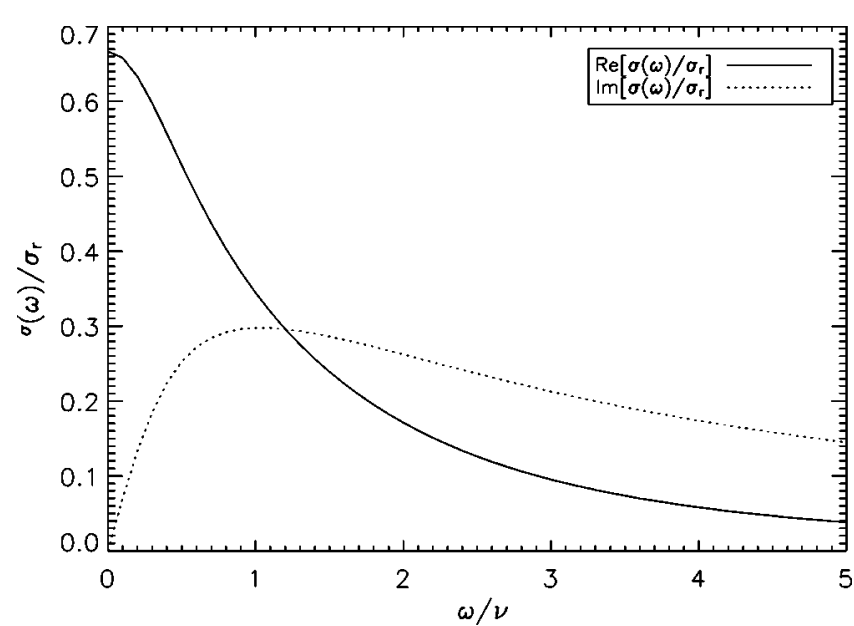

FIG. 1. Real and imaginary parts of $\hat{\sigma} / \sigma_{r}$ in the low frequency range for $\epsilon=0.1$.

$$
\begin{aligned}
& \sum \frac{\gamma_{n}}{\kappa_{n}} \simeq 1-1.48 \sqrt{\epsilon}+\mathcal{O}(\epsilon), \\
& \sum \frac{\gamma_{n}}{\kappa_{n}^{2}} \simeq 1-2 \times 1.48 \sqrt{\epsilon}+\mathcal{O}(\epsilon) .
\end{aligned}
$$

Figure 1 shows the frequency dependence of the electrical conductivity using this expansion.

For high frequencies where $\omega>\nu_{e}$, the $\omega$-dependent factor in the conductivity can be expressed as

$$
\begin{aligned}
\hat{\alpha}_{e}(\omega) \simeq & +\sum \gamma_{n}\left(\kappa_{n}-2\right)-\frac{\nu_{e}^{2}}{\omega^{2}} \sum \gamma_{n} \kappa_{n}-\frac{i \omega}{\nu_{e}}\left[2-\sum \gamma_{n}\right. \\
& \left.+\frac{\nu_{e}^{2}}{\omega^{2}} \sum \gamma_{n}\left(1-2 \kappa_{n}\right)\right] .
\end{aligned}
$$

In this limit, the real part of $\hat{\sigma}(\omega)$ decreases as $\left(\nu_{e} / \omega\right)^{2}$. The imaginary part also decreases, but at a slower rate $\left(\sim \nu_{e} / \omega\right)$, and both asymptote to zero as shown in Fig. 2. Since in Eqs. (48)-(55) a small (or large) $\omega / \nu_{e}$ is considered after a small $\sqrt{\epsilon}$ assumption, what is considered as a small (or large) frequency is to be compared with the magnitude of the field

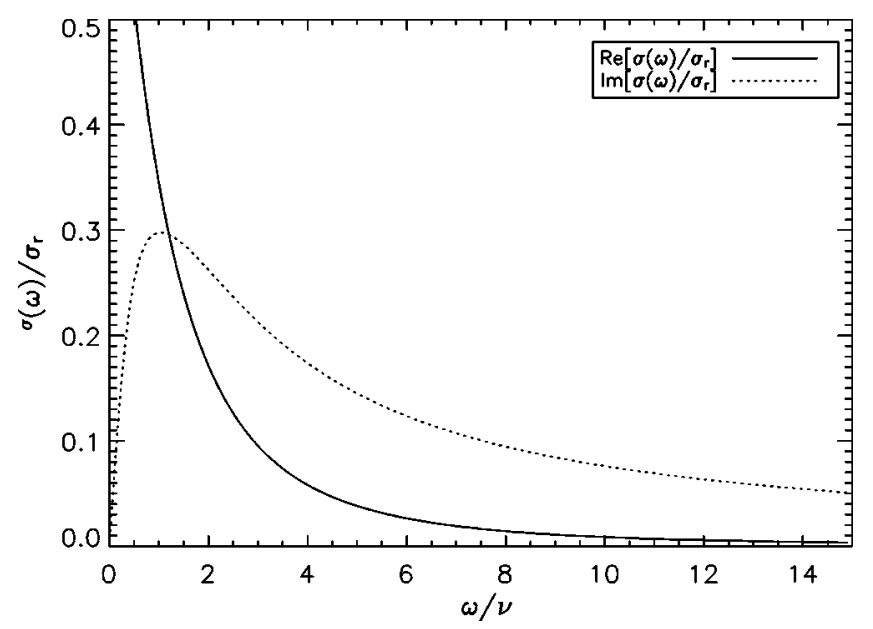

FIG. 2. Asymptotic decay of $\hat{\sigma} / \sigma_{r}$ in the high frequency range for $\epsilon=0.1$.

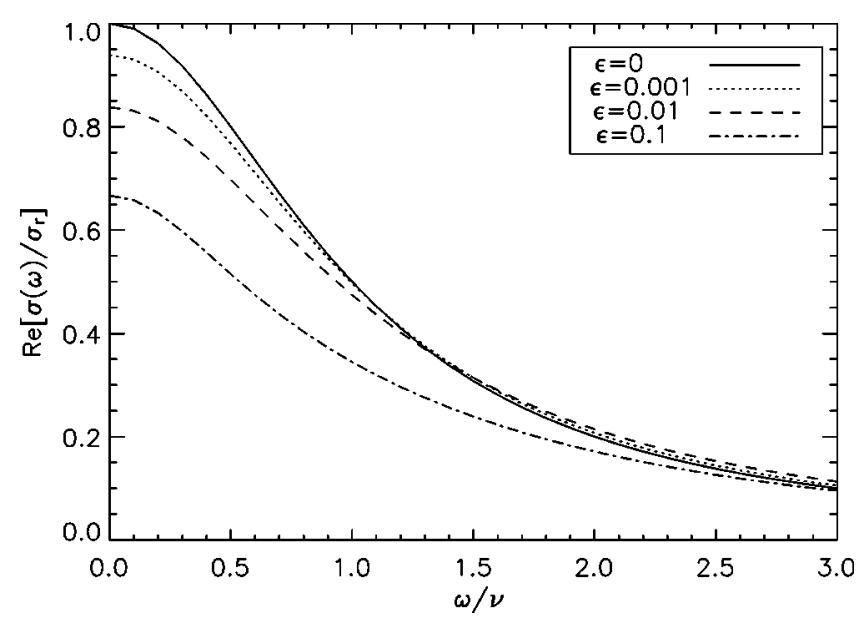

FIG. 3. Real part of $\hat{\sigma} / \sigma_{r}$ for various values of $\epsilon$ as a function of $\omega$.

modulation. That is, the high frequency asymptotic behavior is expected to be seen only for $\omega / \nu_{e} \gg 1 / \sqrt{\epsilon}$.

Note that in the static, low frequency limit, the effects of trapped particles are present for $\omega=0$. On the other hand, as $\omega \rightarrow \infty$ (initial times) there are no significant trapped particle effects. Figures 3 and 4 show the effects of $\epsilon$ on the real and imaginary parts of $\hat{\sigma}(\omega)$. It can be shown, using the estimates in Eqs. (51)-(54), that

$$
\begin{aligned}
& \operatorname{Re}[\hat{\sigma}(\omega)] \sim \begin{cases}1+\mathcal{O}(\sqrt{\epsilon})+\mathcal{O}(\sqrt{\epsilon})\left(\omega / \nu_{e}\right)^{2}, & \omega \ll \nu_{e} \\
{[1+\mathcal{O}(\sqrt{\epsilon})]\left(\nu_{e} / \omega\right)^{2},} & \omega \gg \nu_{e},\end{cases} \\
& \operatorname{Im}[\hat{\sigma}(\omega)] \sim \begin{cases}{[1+\mathcal{O}(\sqrt{\epsilon})] \omega / \nu_{e},} & \omega \ll \nu_{e} \\
{\left[1+\mathcal{O}\left(\epsilon^{3 / 2}\right)\right] \nu_{e} / \omega,} & \omega \gg \nu_{e} .\end{cases}
\end{aligned}
$$

These relations are qualitatively consistent with the behavior shown in Figs. 3 and 4.

\section{FLOW EVOLUTION}

With the closure for the parallel viscous force obtained previously in Eq. (31), the evolution of the parallel flow can

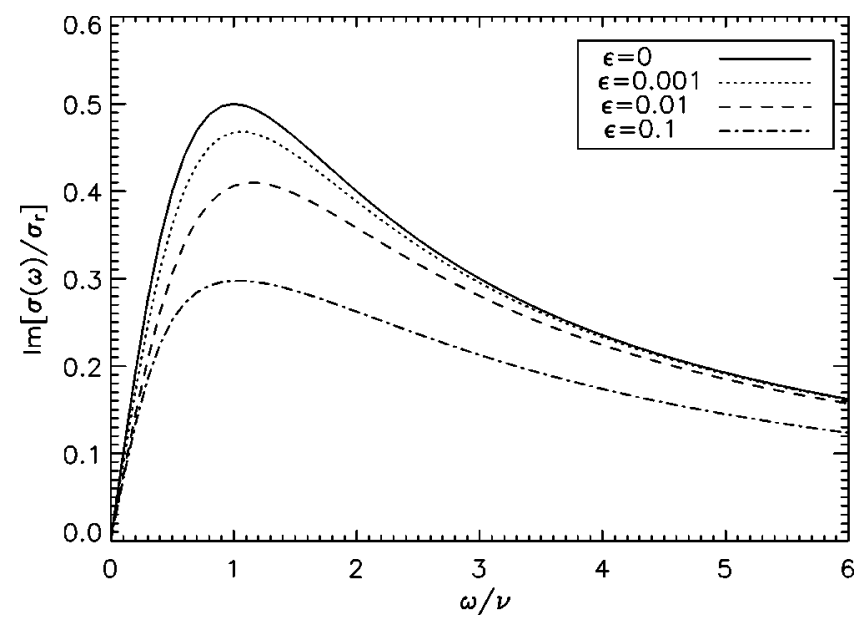

FIG. 4. Imaginary part of $\hat{\sigma} / \sigma_{r}$ for various values of $\epsilon$ as a function of $\omega$. 
be calculated as an initial value problem. Two schemes are considered: the parallel flow damping within the bumpy cylinder model, and an extension to an axisymmetric geometry and thus to the time dynamics of the poloidal flow in a magnetically confined toroidal plasma.

The simplicity of the one-dimensional, bumpy cylinder magnetic field model permits a full calculation of the parallel flow dynamics including effects of initial conditions. Consider the total flux-surface-averaged momentum equation for the plasma

$$
m n\left\langle B^{2}\right\rangle \frac{\partial U(t)}{\partial t}=-\left\langle\mathbf{B} \cdot \boldsymbol{\nabla} \cdot \Pi_{\|}\right\rangle .
$$

Since the time dependence of the left side is of interest, we start by again taking a Laplace transform and work from the full frequency-dependent closure in Eq. (31) as follows

$$
[\hat{v}(\omega)-i \omega] \hat{U}(t)=-\hat{Y}(\omega) .
$$

Considering the small $\sqrt{\epsilon}$ approximation in Eqs. (38) and (39) for $\hat{v}$ and $\hat{Y}$, we have

$$
\begin{aligned}
& \hat{v} \simeq \frac{m}{T} \int d^{3} v \bar{\nu} \frac{v^{2}}{3}\left(1-\frac{i \omega}{\bar{\nu}}\right) \hat{f}_{t} \frac{f_{M}}{n}, \\
& \hat{\Upsilon} \simeq \frac{m}{T} \int d^{3} v \frac{v^{2}}{3} \frac{f_{M}}{n}\left(1-\frac{i \omega}{\bar{\nu}}\right)\left(1+\hat{f}_{t}\right) \hat{f}_{g} .
\end{aligned}
$$

Solving for $\hat{U}$ in Eq. (59) at this point would lead again to infinite sums in the denominator. Instead, the inverse Laplace transform can be taken on both sides and after some manipulation (see Appendix B) one obtains an inhomogeneous integral equation for $U(t)$ :

$$
U(t)=h(t)+\int_{0}^{t} K(t ; \tau) U(\tau) d \tau
$$

This equation gives the time evolution of the parallel flow and has "memory" of the localization of the initial distribution relative to the boundary with trapped particle space. The functions $h(t)$ and $K(t ; \tau)$ in Eq. (62) are defined in Appendix B.

The analysis for the bumpy cylinder can also be employed for a toroidal magnetic field. For the dynamic evolution of the "parallel" flow in an axisymmetric configuration the initial distribution in the pitch-angle variable is not taken into account for simplicity. In this configuration, the magnetic field can be written as

$$
\mathbf{B}=B_{T} \hat{\zeta}+B_{P} \hat{\theta},
$$

where $B_{T}$ and $B_{P}$ are the components of the magnetic field in the toroidal $(\zeta)$ and poloidal $(\theta)$ directions, respectively.

To apply the model developed in the previous sections to this geometry some modifications have to be introduced. In such a configuration, the field modulations along a field line are not unidimensional. That is, the relevant flow variable to be considered is the poloidal flow defined by ${ }^{17}$

$$
U_{\theta}(\psi, t) \equiv \frac{\mathbf{V} \cdot \boldsymbol{\nabla} \theta}{\mathbf{B} \cdot \boldsymbol{\nabla} \theta}=\frac{V_{\|}}{B}+\frac{\mathrm{I}}{B^{2}}\left(\frac{d \phi}{d \psi}+\frac{1}{n q} \frac{d p}{d \psi}\right),
$$

where the first term is due to the parallel flow velocity and the second is due to the perpendicular flows in the plasma, which to lowest order in gyroradius are a combination of the $\mathbf{E} \times \mathbf{B}$ and diamagnetic flows:

$$
\mathbf{V}_{\perp}=\frac{\mathbf{E} \times \mathbf{B}}{B^{2}}+\frac{\mathbf{B} \times \boldsymbol{\nabla} p}{n q B^{2}}
$$

In Eq. (64) we have used $\mathbf{E}=-\boldsymbol{\nabla} \phi$ and $\psi$ is the poloidal magnetic flux function defined by $B_{P} \hat{\theta} \equiv \boldsymbol{\nabla} \zeta \times \nabla \psi$.

From the parallel (to $\mathbf{B}$ ) momentum balance, an evolution equation for $U_{\theta}$ will include a contribution $\left(\propto q^{2}\right)$ from the toroidal flow: ${ }^{5}$

$$
n m\left(1+2 q^{2}\right) \frac{\partial U_{\theta}}{\partial t}=-\frac{\left\langle\mathbf{B} \cdot \boldsymbol{\nabla} \cdot \boldsymbol{\Pi}_{\|}\right\rangle}{\left\langle B_{P}^{2}\right\rangle}+4 \pi\left\langle\frac{\partial \phi^{\prime}}{\partial t}\right\rangle,
$$

where the safety factor is defined as $q=\epsilon B_{T} / B_{p}$ in which now $\epsilon \equiv r / R_{0} \ll 1$ is the inverse aspect ratio.

The closure in Eq. (31) may now be introduced. In this problem parallel stress damps the poloidal component of the parallel flow since toroidal momentum is only damped by the (higher order) perpendicular stress. With the flux surface average in this case being defined as

$$
\langle A\rangle \equiv \oint \frac{d \theta A(\theta)}{\mathbf{B} \cdot \boldsymbol{\nabla} \theta} / \oint \frac{d \theta}{\mathbf{B} \cdot \boldsymbol{\nabla} \theta},
$$

one can write

$$
\left\langle\widehat{\mathbf{B} \cdot \boldsymbol{\nabla} \cdot \Pi_{\|}}\right\rangle=n m\left\langle B^{2}\right\rangle\left[\hat{U}_{\theta} \hat{v}(\omega)+U_{\theta 0}\right] .
$$

Once again, Eqs. (60) and (61) can be used to obtain an approximate closure to lowest order in $\hat{f}_{t}$. Introducing this closure in Eq. (66) yields

$$
\begin{aligned}
& {\left[\frac{\left\langle B^{2}\right\rangle}{\left\langle B_{P}^{2}\right\rangle} \hat{v}(\omega)-i \omega\left(1+2 q^{2}\right)\right] \hat{U}_{\theta}=\left[\left(1+2 q^{2}\right)-\frac{\left\langle B^{2}\right\rangle}{\left\langle B_{P}^{2}\right\rangle}\right] U_{\theta 0}} \\
& \quad+\frac{4 \pi}{n m}\left\langle\frac{\partial \phi^{\prime}}{\partial t}\right\rangle,
\end{aligned}
$$

and taking the inverse Laplace transform on both sides yields the integral equation

$$
U_{\theta}(t)=h_{\theta}(t)+\int_{0}^{t} K_{\theta}(t ; \tau) U_{\theta}(t) d \tau,
$$

where $h_{\theta}(t)$ and $K_{\theta}(t ; \tau)$ are defined in Appendix B. Using the approximation described in Sec. VI, the asymptotic limits of the poloidal flow evolution for short and long times can be inspected. For $t \ll 1 / \bar{\nu}$, Eq. (70) can be roughly approximated by

$$
U_{\theta}(t) \simeq t K_{\theta}(t ; t) U_{\theta}(t),
$$

where we have assumed the initial condition is already damped. Then the characteristic damping time is initially given by $\tau_{p} \simeq 1 / K_{\theta}(t ; t)$ which yields (see Appendix B) 


$$
1 / \tau_{p} \simeq \frac{0.51}{\epsilon} \frac{m}{T} \int d^{3} v \bar{\nu} \frac{v^{2}}{3} \frac{f_{M}}{n} .
$$

The numerical result in Eq. (72) is similar to the estimate for the damping rate in Ref. 8 for this limit. This result is also obtained in Ref. 10 when taking a time average including the transient behavior due to the initial perturbation.

In the present $\sqrt{\epsilon} \ll 1$ expansion, in addition to the damping rate $\nu_{p}$ there is a small oscillatory $\left(\omega_{r}\right)$ response. $^{8}$ It can be recovered by writing $i \omega=\nu_{p}+i \omega_{r}$ and expanding $v(\omega)$ about the damping rate assuming $\nu_{p} \gg \omega_{r}$. By taking account of the Landau-type pole ${ }^{8}$ in the denominator of $f_{c}$ and equating the imaginary parts one obtains an imaginary component of the frequency $\omega_{r}$. This effect contributes slight oscillatory responses in $\left\langle\mathbf{B} \cdot \boldsymbol{\nabla} \cdot \Pi_{\|}\right\rangle$and in the kernels of the time-history integrals in Eq. (70). This feature of the flow evolution is neglected in this paper because we are concentrating on the dominant, damping effect of the parallel viscosity.

For long times, in recovering the static limit, one can rewrite the flow evolution as

$$
U_{\theta}(t) \simeq U_{\theta}(t) \int_{0}^{t} K_{\theta}(t ; \tau) d \tau
$$

and thus, the characteristic damping rate is obtained from

$$
\int_{0}^{t} K_{\theta}(t ; \tau) d \tau=1
$$

In the small $\epsilon$ approximation the damping rate $\nu_{p}$ for the static calculation is obtained (see Appendix B):

$$
\nu_{p} \simeq \frac{0.31}{\sqrt{\epsilon}} \frac{m}{T} \int d^{3} v \bar{\nu} \frac{v^{2}}{3} \frac{f_{M}}{n} .
$$

This result clearly violates the static assumption, as pointed out by other authors (see, for example, Refs. 5 and 9).

\section{SUMMARY}

A simple inhomogeneous magnetic field model has been used to calculate the time-dependent kinetic closure for the parallel viscous force $\left\langle\mathbf{B} \cdot \boldsymbol{\nabla} \cdot \boldsymbol{\Pi}_{\|}\right\rangle$in the banana collisionality regime. This model retains the effect of trapped particles and can be extended to more complicated geometries.

The calculation was carried out using the ChapmanEnskog-like approach developed by Wang and Callen. ${ }^{13}$ However, the same results should be able to be obtained using the standard approach in neoclassical theory. The same frequency-dependent closure was obtained before using the usual method while not considering initial conditions. ${ }^{6,8}$ By treating Eq. (3) in Ref. 8 as an initial value problem and taking a formal Laplace transform, the time-dependent closure [Eq. (41)] should be obtained (in the large aspect ratio case) when the inverse transform is calculated. Also, Shaing showed the equivalence of this method and the traditional one in Ref. 4 for the static case in Ref. 18.

In carrying out the calculation, a formal Laplace transform is introduced which retains the initial value character of the problem. Further analytic progress can be made by employing an expansion for small field modulations. In such a case, the inverse Laplace transform can be calculated to any order both analytically and numerically. The key result, Eq. (41), is an explicit time-dependent closure for the parallel viscous force which may be useful in numerical codes as well as for the theoretical modeling of fast phenomena $(t$ $\lesssim 1 / \nu)$.

The evolution of the driven parallel/poloidal flow in the expressions obtained are described by integral equations which are still to be evaluated or solved. The results obtained here have various applications that are yet to be exploited. For example, they can be used to explore the dynamics of the transition of the perpendicular dielectric from regular Alfvénic to enhanced neoclassical regimes ${ }^{19}$ and for the effects of flow dynamics on neoclassical tearing modes. ${ }^{20}$

Extension of these various results to include heat flux effects are given in Appendix B. These extensions are required to obtain net electrical conductivity and poloidal flow damping effects that are not just correct to within about a factor of 2 , but instead are within about the $1 / \ln \Lambda \sim 7 \%$ intrinsic accuracy of the Coulomb collision operator.

\section{ACKNOWLEDGMENTS}

The authors wish to thank Dr. Eric Held for helpful discussions. This research was supported by U. S. Department of Energy under Grant No. DE-FG02-86ER53218.

\section{APPENDIX A: CLOSURE FOR $\left\langle\widehat{\left.\overline{B \cdot \nabla \cdot \Pi_{\|}}\right\rangle}\right.$}

In this Appendix, the closure given in Eq. (31) is obtained from the solution for $\hat{g}_{c}$ obtained in Sec. V. To simplify the notation we introduce the drive $\hat{D}(v, i \omega)$ defined as

$$
\begin{aligned}
\hat{D}(v, i \omega) \equiv & \frac{f_{M}}{p}\left\langle\widehat{\mathbf{B} \cdot \boldsymbol{\nabla} \cdot \Pi_{\|}}\right\rangle-\frac{m}{T}\left\langle B^{2}\right\rangle f_{M}\left(i \omega \hat{U}+U_{0}\right) \\
& +\frac{1}{v}\left\langle B^{2}\right\rangle\left[C^{1}(\hat{V})+\bar{\nu} \hat{V}\right],
\end{aligned}
$$

which contains the $\lambda$-independent terms on the right side of Eq. (21). Using Eqs. (26) and (27), the speed dependence in the expansion for $\hat{g}_{c}$ can be written as

$$
\hat{Y}_{n}=-\frac{1}{\bar{\nu}}\left[\frac{\eta_{n} \hat{D}}{\kappa_{n}-i \omega / \bar{\nu}}+\frac{m}{T} f_{M} \frac{\alpha_{n}}{\kappa_{n}-i \omega / \bar{\nu}}\right] .
$$

The coefficients $\eta_{n}$ and $\alpha_{n}$ are calculated from

$$
\begin{gathered}
\eta_{n}=\frac{\int_{0}^{\lambda_{c}} \Lambda_{n} d \lambda}{\int_{0}^{\lambda_{c}} \Lambda_{n}^{2} \frac{\partial}{\partial \lambda}\langle\sqrt{1-\lambda B}\rangle d \lambda}, \\
\alpha_{n}=\frac{\int_{0}^{\lambda_{c}} d \lambda\left\langle\frac{B}{v_{\|}}\right\rangle g_{0}(\lambda) \Lambda_{n}}{\int_{0}^{\lambda_{c}} \Lambda_{n}^{2} \frac{\partial}{\partial \lambda}\langle\sqrt{1-\lambda B}\rangle d \lambda} .
\end{gathered}
$$

The flow variable $\hat{V}$, given by its definition in Eq. (24), is thus 


$$
\hat{V}=\frac{v}{\bar{\nu}} \frac{1}{\left\langle B^{2}\right\rangle}\left(\hat{f}_{c} \hat{D}+\left\langle B^{2}\right\rangle^{1 / 2} f_{M} \frac{m}{T} \hat{f}_{g}\right)
$$

where the definitions for $\hat{f}_{c}$ and $\hat{f}_{g}$ are those in Eqs. (29) and (30) where

$$
\gamma_{n}=-\frac{3}{4}\left\langle B^{2}\right\rangle \eta_{n} \int_{0}^{\lambda_{c}} d \lambda \Lambda_{n}
$$

and

$$
\chi_{n}=-\frac{3}{4}\left\langle B^{2}\right\rangle^{1 / 2} \alpha_{n} \int_{0}^{\lambda_{c}} d \lambda \Lambda_{n}
$$

Substituting the drive $\hat{D}$ from Eq. (A1) in Eq. (A5) we obtain a relation between $\hat{V}$ and $\left\langle\widehat{\mathbf{B} \cdot \boldsymbol{\nabla} \cdot \Pi_{\|}}\right\rangle$:

$$
\begin{aligned}
\frac{\bar{\nu}}{v} \frac{T}{m} \hat{V}\left(\frac{1}{\hat{f}_{c}}-1\right)= & \frac{f_{M}}{n m}\left\langle B^{2}\right\rangle^{-1}\left\langle\widehat{\mathbf{B} \cdot \boldsymbol{\nabla} \cdot \Pi_{\|}}\right\rangle-\frac{C(\hat{V})}{v}-(i \omega \hat{U} \\
& \left.+U_{0}+\left\langle B^{2}\right\rangle^{-1 / 2} \frac{\hat{f}_{g}}{\hat{f}_{c}}\right) f_{M} .
\end{aligned}
$$

Calculating the momentum moment of Eq. (A8) and using the momentum conserving property of the collision operator, $\int d^{3} v v C^{1}(\hat{V})=0$, we obtain

$$
\begin{aligned}
\left\langle\widehat{\mathbf{B} \cdot \boldsymbol{\nabla} \cdot \Pi_{\|}}\right\rangle= & m n\left\langle B^{2}\right\rangle\left\{\int d^{3} v \frac{v}{3} \bar{\nu} \hat{V}\left(\frac{1}{\hat{f}_{c}}-1\right)+i \omega \hat{U}+U_{0}\right. \\
& \left.-\left\langle B^{2}\right\rangle^{-1 / 2} \frac{m}{T} \int d^{3} v \frac{v^{2}}{3} \frac{f_{M}}{n} \frac{\hat{f}_{g}}{\hat{f}_{c}}\right\}
\end{aligned}
$$

To find a relation between $\hat{V}$ and $\hat{U}$, we assume that $\hat{V}$ can be expanded in Laguerre polynomials $\left(L_{0}^{3 / 2}=1, L_{0}^{3 / 2}=5 / 2\right.$ $\left.-m v^{2} / 2 T, \ldots\right)$ as $^{8}$

$$
\hat{V}(v)=\frac{m}{T} \frac{f_{M}}{n} v \sum_{n} \hat{V}_{n} L_{n}^{3 / 2}
$$

Using the constraint that the parallel momentum moment of $\hat{F}_{0}$ has to vanish one can obtain $\hat{V}_{0}=\hat{U} n$. This is the only relevant term in the approximation considered here since we are neglecting higher order terms in $L_{n}^{3 / 2}$. Introducing this relation in Eq. (A9), the closure given in Eq. (31) is obtained.

\section{APPENDIX B: INVERSE LAPLACE TRANSFORMS}

In this Appendix the inverse Laplace transform of the frequency-dependent closure and the parallel and poloidal flow evolution equations are developed in some detail. For the parallel stress, the term in curly brackets in Eq. (40) can be inverted term by term:

$$
L^{-1}\left\{\hat{f}_{t}\right\}=\sum\left(\kappa_{n}-1\right) \bar{\nu} \gamma_{n} e^{-\kappa_{n} \bar{\nu} t}+\left(1-\sum \gamma_{n}\right) \delta(t)
$$

Defining the integral $I_{n}(t)=\int_{0}^{t} d \tau e^{-\kappa_{n} \bar{\nu}(t-\tau)} U(\tau)$, the convolution with the parallel flow variable is

$$
L^{-1}\left\{\hat{f}_{t} \hat{U}\right\}=\left(1-\sum \gamma_{n}\right) U(t)+\bar{\nu} \sum \gamma_{n}\left(\kappa_{n}-1\right) I_{n}(t),
$$

and the triple convolution can be calculated as follows:

$$
\begin{aligned}
L^{-1}\left\{-\frac{i \omega}{\bar{\nu}} \hat{f}_{t} \hat{U}\right\}= & \frac{1}{\bar{\nu}}\left(1-\sum \gamma_{n}\right)\left(\frac{\partial U(t)}{\partial t}+U_{0} \delta(t)\right) \\
& +\sum \gamma_{n}\left(\kappa_{n}-1\right)\left[U(t)-\kappa_{n} \bar{\nu} I_{n}(t)\right] .
\end{aligned}
$$

Putting these results together we have

$$
\begin{aligned}
L^{-1}\left\{\frac{\hat{f}_{t}}{\hat{f}_{c}} \hat{U}\right\} \simeq & \frac{1}{\bar{\nu}}\left(1-\sum \gamma_{n}\right)\left(\frac{\partial U(t)}{\partial t}+U_{0} \delta(t)\right)+[1 \\
& \left.+\sum \gamma_{n}\left(\kappa_{n}-2\right)\right] U(t)-\sum \gamma_{n}\left(\kappa_{n}\right. \\
& -1)^{2} \bar{\nu} I_{n}(t)
\end{aligned}
$$

which, after some manipulation, leads to the dynamic closure in Eq. (41) for $t>0$.

To obtain the time evolution of the parallel flow variable, the inverse Laplace transform is calculated on both sides of Eq. (59). For the right side, using Eq. (39) and the previous results one can obtain

$$
\begin{aligned}
L^{-1}\left\{\frac{\hat{f}_{g}}{\hat{f}_{c}}\right\} \simeq & \sum_{m, n}\left\{\delta(t) \gamma_{n} \chi_{m}-\bar{\nu}\left(\kappa_{m}-1\right) e^{-\kappa_{m} \bar{\nu} t}\left[\left(\chi_{m} \gamma_{n}\right.\right.\right. \\
& \left.\left.+\chi_{n} \gamma_{m}\right)-\bar{\nu} \chi_{m} \gamma_{n}\left(\kappa_{n}-1\right) \int_{0}^{t} e^{\bar{\nu} \tau\left(\kappa_{m}-\kappa_{n}\right)} d \tau\right] .
\end{aligned}
$$

To eliminate the $\delta$ functions, a time integral is taken on both sides and after a simple calculation one can solve for $U(t)$. Equation (62) is obtained for which we define the inhomogeneous term and the integration kernel as follows:

$$
\begin{aligned}
h(t)= & \frac{1}{\left(\sum \gamma_{m}-2\right)} \int d^{3} v \frac{m}{T} \frac{v^{2}}{3} \frac{f_{M}}{n} \sum_{m, n}\left\{\frac{\chi_{n} \gamma_{m}}{\kappa_{m} \kappa_{n}}-\left(\kappa_{m}\right.\right. \\
& -1) \frac{\chi_{m} \gamma_{n}}{\kappa_{m}} e^{-\kappa_{m} \bar{\nu} t}\left[\left(1+\frac{\chi_{n} \gamma_{m}}{\kappa_{n} \chi_{m} \gamma_{n}}\right)-\bar{\nu}\left(\kappa_{n}\right.\right. \\
& \left.\left.-1) \int_{0}^{t} e^{\bar{\nu} \tau\left(\kappa_{m}-\kappa_{n}\right)} d \tau\right]\right\}, \\
K(t ; \tau)= & \frac{1}{\left(\sum \gamma_{m}-2\right)} \int d^{3} v \bar{\nu} \frac{m}{T} \frac{v^{2}}{3} \frac{f_{M}}{n}\left\{1+\sum \frac{\gamma_{m}}{\kappa_{m}}\left[\left(\kappa_{m}\right.\right.\right. \\
& \left.\left.-1)^{2} e^{-\kappa_{m} \bar{\nu}(t-\tau)}-1\right]\right\} .
\end{aligned}
$$

For the toroidal geometry, the inverse Laplace transform of each term in Eq. (69) is calculated as before. A time integral has to be calculated in this case also and one can obtain Eq. (70) where the inhomogeneous term and kernel are defined by

$$
h_{\theta}(t)=a\left[\left(1+2 q^{2}-\frac{\left\langle B^{2}\right\rangle}{\left\langle B_{P}^{2}\right\rangle}\right) U_{\theta 0}-\frac{4 \pi}{n m}\left\langle\phi^{\prime}(t)-\phi_{0}^{\prime}\right\rangle\right],
$$




$$
\begin{aligned}
K_{\theta}(t ; \tau)= & -a \frac{\left\langle B^{2}\right\rangle}{\left\langle B_{P}^{2}\right\rangle} \frac{m}{T} \int d^{3} v \bar{\nu} \frac{v^{2}}{3} \frac{f_{M}}{n}\left\{1-\sum \frac{\gamma_{n}}{\kappa_{n}}\left[1+\left(\kappa_{n}\right.\right.\right. \\
& \left.\left.-1)^{2} e^{-\kappa_{n} \bar{\nu}(t-\tau)}\right]\right\},
\end{aligned}
$$

and the factor $a$ for both expressions is

$$
a=\left[1+2 q^{2}+\frac{\left\langle B^{2}\right\rangle}{\left\langle B_{P}^{2}\right\rangle}\left(1-\sum \gamma_{n}\right)\right]^{-1} \simeq \frac{\left\langle B^{2}\right\rangle}{\left\langle B_{P}^{2}\right\rangle}\left(1-\sum \gamma_{n}\right),
$$

where the second line is valid for small $\epsilon$. Using Eq. (B14) and the values in Eqs. (51)-(54), the kernel of integration $K_{\theta}(t ; t) \sim 1 / \tau_{p}$ for the $t \ll \bar{\nu}$ case in Sec. VII is

$$
K_{\theta}(t ; t) \simeq\left(\frac{0.51}{\epsilon}-2.69\right) \frac{m}{T} \int d^{3} v \bar{\nu} \frac{v^{2}}{3} \frac{f_{M}}{n} .
$$

In the long time case, for the static limit at the end of Sec. VII we have, for $\int_{0}^{t} K_{\theta}(t ; \tau) d \tau=1$,

$$
t-\sum \frac{\gamma_{n}}{\kappa_{n}}\left[t+\int_{0}^{t}\left(\kappa_{n}-1\right)^{2} e^{-\kappa_{n} \bar{\nu}(t-\tau)} d \tau\right]=1-\sum \gamma_{n},
$$

or, solving for $t=\tau_{p}$,

$$
\tau_{p} \simeq\left(\frac{\sqrt{\epsilon}}{0.31}\right) / \frac{m}{T} \int d^{3} v \bar{\nu} \frac{v^{2}}{3} \frac{f_{M}}{n},
$$

which yields the damping rate in Eq. (75) and the factor $a$ for both expressions, which is

$$
a=\left[1+2 q^{2}+\frac{\left\langle B^{2}\right\rangle}{\left\langle B_{P}^{2}\right\rangle}\left(1-\sum \gamma_{n}\right)\right]^{-1} \simeq \frac{\left\langle B^{2}\right\rangle}{\left\langle B_{P}^{2}\right\rangle}\left(1-\sum \gamma_{n}\right),
$$

where the second line is valid for small $\epsilon$. Using Eq. (B14) and the values in Eqs. (51)-(54), the kernel of integration $K_{\theta}(t ; t) \sim 1 / \tau_{p}$ for the $t \ll \bar{\nu}$ case in Sec. VI is

$$
K_{\theta}(t ; t) \simeq\left(\frac{0.51}{\epsilon}-2.69\right) \frac{m}{T} \int d^{3} v \bar{\nu} \frac{v^{2}}{3} \frac{f_{M}}{n} .
$$

In the long time case, for the static limit at the end of Sec. VII we have, for $\int_{0}^{t} K_{\theta}(t ; \tau) d \tau=1$,

$$
t-\sum \frac{\gamma_{n}}{\kappa_{n}}\left[t+\int_{0}^{t}\left(\kappa_{n}-1\right)^{2} e^{-\kappa_{n} \bar{\nu}(t-\tau)} d \tau\right]=1-\sum \gamma_{n},
$$

or, solving for $t=\tau_{p}$,

$$
\tau_{p} \simeq\left(\frac{\sqrt{\epsilon}}{0.31}\right) / \frac{m}{T} \int d^{3} v \bar{\nu} \frac{v^{2}}{3} \frac{f_{M}}{n},
$$

\section{APPENDIX C: HEAT FLUX EFFECTS}

To include heat flux effects, the distribution function to be considered in the Chapman-Enskog procedure is an equilibrium flow and heat-flux shifted Maxwellian plus a small kinetic distortion:

$$
f=f_{M}\left[1+\frac{m}{T} \mathbf{v} \cdot\left(\frac{2}{5 n T} \mathbf{q}\right) L_{1}^{3 / 2}\right]+F,
$$

where $f_{M}$ is defined in Eq. (2). In this case, the recast DKE includes two extra terms and, upon defining a parallel heatflux variable $Q(\psi)=\mathbf{q} \cdot \mathbf{B} / B^{2}$ to satisfy heat-flux incompressibility, can be written as ${ }^{17}$

$$
\begin{aligned}
\frac{d F}{d t} & +v_{\|} \mathbf{b} \cdot \boldsymbol{\nabla}\left[F+\frac{m}{T} v_{\|} B\left(U-\frac{2}{5 p} Q L_{1}^{3 / 2}\right) f_{M}\right]=C(F) \\
& +\frac{v_{\|}}{p} f_{M}\left(\mathbf{b} \cdot \boldsymbol{\nabla} \cdot \Pi_{\|}+\frac{2}{5} L_{1}^{3 / 2} \mathbf{b} \cdot \boldsymbol{\nabla} \cdot \Theta_{\|}\right) .
\end{aligned}
$$

In Eqs. (C1) and (C2), $L_{1}^{3 / 2}=5 / 2-x$ is the Laguerre polynomial in the usual variable $x=v^{2} / v_{t}^{2}$ and the heat stress tensor is given by

$$
\Theta=\int d^{3} v m\left(\mathbf{v v}-\frac{1}{3} v^{2} \mathbf{I}\right) L_{1}^{3 / 2} f .
$$

Solving Eq. (C2) to lowest order as done previously in Sec. IV the lowest order distortion is obtained:

$$
F_{0}=-\frac{m}{T} v_{\|} B\left(U-\frac{2}{5 p} Q L_{1}^{3 / 2}\right) f_{M}+g(v, \lambda, \varsigma, t) .
$$

Calculating the annihilator defined in Eq. (20) in the next order DKE and taking a Laplace transform as before, one obtains a differential equation for $\hat{g}$ for circulating particles similar to Eq. (21). The $\lambda$-independent drives in the source now include extra terms due to the heat-flux contribution

$$
\begin{aligned}
\hat{D}(v, i \omega) \equiv & \left(\left\langle\widehat{\mathbf{B} \cdot \boldsymbol{\nabla} \cdot \Pi_{\|}}\right\rangle L_{0}^{3 / 2}+\frac{2}{5}\left\langle\widehat{\mathbf{B} \cdot \boldsymbol{\nabla} \cdot \Theta_{\|}}\right\rangle L_{1}^{3 / 2}\right) f_{M} \\
& +\frac{1}{v}\left\langle B^{2}\right\rangle\left[C^{1}\left(\hat{g}_{1}\right)+\bar{\nu} \hat{g}_{1}\right]-\frac{m}{T}\left\langle B^{2}\right\rangle f_{M}\left[i \omega \left(\hat{U} L_{0}^{3 / 2}\right.\right. \\
& \left.\left.-\frac{2}{5 p} \hat{Q} L_{1}^{3 / 2}\right)+\left(U_{0} L_{0}^{3 / 2}-\frac{2}{5 p} Q_{0} L_{1}^{3 / 2}\right)\right] . \quad(\mathrm{C} 5)
\end{aligned}
$$

Thus, the structure of the solution for the integration constant in the lowest order solution in the same as before [Eq. (28)]. The extra terms appear when one substitutes the drive $\hat{D}$ given by Eq. (C5) into Eq. (A5).

In this case, in order to obtain the closures for $\left\langle\widehat{\mathbf{B} \cdot \nabla \cdot \Pi_{\|}}\right\rangle$ and $\left\langle{\widehat{\mathbf{B} \cdot \boldsymbol{\nabla} \cdot \boldsymbol{\Theta}_{\|}}}_{\|}\right.$we generalize the conditions used before by requiring

$$
\int d^{3} v v L_{i}^{3 / 2} C^{1}(\hat{V})=0
$$

and 


$$
\int d^{3} v L_{i}^{3 / 2} v_{\|} \hat{F}=0
$$

for $i=0,1$. Proceeding as in Appendix A and calculating one more term in the expansion for $\hat{V}$, namely, $\hat{V}_{1}=-(2 / 5 T) \hat{Q}$, one can show that the closures are given by

$$
\left\langle\widehat{\mathbf{B} \cdot \boldsymbol{\nabla} \cdot \Pi_{\|}}\right\rangle=n m\left\langle B^{2}\right\rangle\left(\hat{U} \hat{v}_{00}-\frac{2}{5 p} \hat{Q} \hat{v}_{01}+U_{0}+\hat{\Upsilon}_{0}\right),
$$

and

$$
\left\langle\widehat{\mathbf{B} \cdot \boldsymbol{\nabla} \cdot \boldsymbol{\Theta}_{\|}}\right\rangle=n m\left\langle B^{2}\right\rangle\left(\hat{U} \hat{v}_{01}-\frac{2}{5 p} \hat{Q} \hat{v}_{11}-\frac{1}{p} Q_{0}+\hat{\Upsilon}_{1}\right) .
$$

Here $\hat{v}_{i j}(\omega)$ are the "viscosity coefficients" [ for $\hat{v}_{i j}(0)=\mu_{i j}$ numerical values see Ref. 17]. Both $\hat{v}_{i j}(\omega)$ and $\hat{\Upsilon}_{i}(\omega)$ are obvious generalizations of the coefficients defined in Eqs. (32) and (34):

$$
\begin{aligned}
& \hat{v}_{i j}(\omega)=\frac{m}{T} \int d^{3} v \bar{\nu} \frac{v^{2}}{3} \frac{f_{M}}{n} L_{i}^{3 / 2} L_{j}^{3 / 2} \frac{\hat{f}_{t}}{\hat{f}_{c}}, \\
& \hat{\Upsilon}_{i}(\omega)=\frac{m}{T} \int d^{3} v \frac{v^{2}}{3} \frac{f_{M}}{n} L_{i}^{3 / 2} \frac{\hat{f}_{g}}{\hat{f}_{c}} .
\end{aligned}
$$

Thus, the frequency dependent closure, including heatflux effects, can be expressed as a matrix equation as follows:

$$
\begin{aligned}
{\left[\begin{array}{c}
\left\langle\widehat{\mathbf{B} \cdot \boldsymbol{\nabla} \cdot \Pi_{\|}}\right\rangle \\
\left\langle\widehat{\mathbf{B} \cdot \boldsymbol{\nabla} \cdot \Theta_{\|}}\right\rangle
\end{array}\right]=} & n m\left\langle B^{2}\right\rangle\left[\begin{array}{ll}
v_{00} & v_{01} \\
v_{01} & v_{11}
\end{array}\right]\left[\begin{array}{c}
\hat{U} \\
-\frac{2}{5 p} \hat{Q}
\end{array}\right] \\
& {\left[\begin{array}{c}
U_{0}+\Upsilon_{0}(\omega) \\
-\frac{1}{p} Q_{0}+\Upsilon_{1}(\omega)
\end{array}\right] . }
\end{aligned}
$$

In order to obtain the heat-flux correction to expression (43), we use the moment approach. ${ }^{4}$ This is, taking the momentum and heat-flux moments (i.e., $m \int d^{3} v v_{\|} L_{i}^{3 / 2}$ for $i=1,2$ ) of the kinetic equation yields a matrix equation for the evolution of $V_{\|}$and $q_{\|}$. The parallel component of these balance equations for electrons can be written in matrix form as

$$
\begin{aligned}
\frac{d}{d t}\left[\begin{array}{c}
V_{\|} B \\
-q_{\|} B / p
\end{array}\right]= & -\frac{e}{m_{e}}\left[\begin{array}{c}
E_{\|} B \\
0
\end{array}\right]-\nu_{e}\left[\mathbb{L}_{]}\left[\begin{array}{c}
V_{\|} B \\
-2 q_{\|} B / 5 p
\end{array}\right]\right. \\
& +\frac{1}{n_{e} m_{e}}\left[\begin{array}{l}
\mathbf{B} \cdot \boldsymbol{\nabla} \cdot \Pi_{\| e} \\
\mathbf{B} \cdot \boldsymbol{\nabla} \cdot \mathrm{\Theta}_{\| e}
\end{array}\right],
\end{aligned}
$$

where $L_{d}$ denotes the $2 \times 2$ matrix containing the usual ${ }^{4}$ electron friction coefficients $\mathrm{L}_{i j}^{e}$. When a bounce average is cal- culated and the closure $(\mathrm{C} 12)$ is introduced, the Laplace transform of Eq. (C13) can be written as

$$
\left[\begin{array}{c}
\left\langle E_{\|} B\right\rangle \\
0
\end{array}\right] \simeq \frac{m_{e} \nu_{e}}{n_{e} e^{2}}[\mathbb{F}]\left[\begin{array}{c}
\left\langle J_{\|} B\right\rangle \\
\frac{2 e}{5 T_{e}}\left\langle q_{\|} B\right\rangle
\end{array}\right]
$$

where we defined

$$
[\mathbb{F}]=\left[L_{1}\right]+\left[\begin{array}{cc}
\left(v_{00}-i \omega\right) / \nu_{e} & v_{01} / \nu_{e} \\
v_{01} / \nu_{e} & \left(v_{11}-5 i \omega / 2\right) / \nu_{e}
\end{array}\right] .
$$

Thus, the electrical conductivity in this more precise approximation can be calculated as

$$
\hat{\sigma}(\omega) \simeq\left(\frac{\mathbb{F}_{22}}{\mathbb{F}_{11} \mathbb{F}_{22}-\mathbb{F}_{10}^{2}}\right) \sigma_{r} .
$$

For the parallel flow damping correction, we generalize the quantities in Eqs. (B6) and (B7) by introducing $L_{i}^{3 / 2}$ [for $\left.h(t) \rightarrow h_{i}(t)\right]$ and $L_{i}^{3 / 2} L_{j}^{3 / 2}\left[\right.$ for $\left.K(t ; \tau) \rightarrow K_{i j}(t ; \tau)\right]$ in the velocity space integrals. Doing so and proceeding as in the last part of Appendix B one can write the evolution for the flow including heat flux effects as an integral (matrix) equation as follows

$$
\left[\begin{array}{c}
U(t) \\
-\frac{1}{p} Q(t)
\end{array}\right]=\left[\begin{array}{l}
h_{0} \\
h_{1}
\end{array}\right]+\int_{0}^{t}\left[\begin{array}{ll}
K_{00} & K_{01} \\
K_{10} & K_{11}
\end{array}\right]\left[\begin{array}{c}
U(\tau) \\
-\frac{2}{5 p} Q(\tau)
\end{array}\right] d \tau .
$$

${ }^{1}$ F. L. Hinton and C. Oberman, Nucl. Fusion 9, 319 (1969).

${ }^{2}$ M. N. Rosenbluth, R. D. Hazeltine, and F. L. Hinton, Phys. Fluids 15, 116 (1972).

${ }^{3}$ F. L. Hinton and M. N. Rosenbluth, Phys. Fluids 16, 836 (1973).

${ }^{4}$ S. P. Hirshman and D. J. Sigmar, Nucl. Fusion 21, 1079 (1981).

${ }^{5}$ S. P. Hirshman, Nucl. Fusion 18, 917 (1978).

${ }^{6}$ K. C. Shaing and S. P. Hirshman, Phys. Fluids B 1, 705 (1989).

${ }^{7}$ M. Taguchi, Plasma Phys. Controlled Fusion 33, 859 (1991).

${ }^{8}$ C. T. Hsu, K. C. Shaing, and R. Gormley, Phys. Plasmas 1, 132 (1994).

${ }^{9}$ R. C. Morris, M. G. Haines, and R. J. Hastie, Phys. Plasmas 3, 4513 (1996).

${ }^{10}$ F. L. Hinton and M. N. Rosenbluth, Plasma Phys. Controlled Fusion 41, 653 (1999).

${ }^{11}$ J. G. Cordey, Nucl. Fusion 16, 499 (1976).

${ }^{12}$ S. Von Goeler, W. Stodiek, and N. Sauthoff, Phys. Rev. Lett. 33, 1201 (1974).

${ }^{13}$ J. P. Wang and J. D. Callen, Phys. Fluids B 4, 1139 (1992).

${ }^{14} \mathrm{~S}$. Chapman and T. G. Cowling, The Mathematical Theory of NonUniform Gases (Cambridge University Press, Cambridge, 1939).

${ }^{15}$ S. P. Hirshman and D. J. Sigmar, Phys. Fluids 10, 153 (1976).

${ }^{16}$ M. N. Rosenbluth, W. M. MacDonald, and D. L. Judd, Phys. Rev. 107, 1 (1957).

${ }^{17}$ J. P. Wang and J. D. Callen, Phys. Fluids B 5, 3207 (1993).

${ }^{18}$ K. C. Shaing, Phys. Fluids 31, 8 (1988).

${ }^{19}$ J. D. Callen, W. X. Qu, K. D. Siebert, B. A. Carreras, K. C. Shaing, and D. A. Spong, Plasma Physics and Controlled Nuclear Fusion Research 1986 (IAEA, Vienna, 1987), Vol. 2, p. 157.

${ }^{20}$ H. R. Wilson, J. W. Connor, R. J. Hastie, and C. C. Hegna, Phys. Plasmas 3, 2 (1996). 